\section{Ocupação e câncer da cavidade oral e orofaringe}

\author{
Occupational status and cancer \\ of the oral cavity and oropharynx
}

\author{
1 Coordenação de Vigilância \\ Sanitária, Secretaria \\ Municipal de Saúde \\ São Paulo, Brasil. \\ 2 Faculdade de Saúde \\ Pública, Universidade de \\ São Paulo, São Paulo, Brasil. \\ 3 Fundação Jorge Duprat \\ Figueiredo de Segurança \\ e Medicina do Trabalho, \\ São Paulo, Brasil. \\ 4 Faculdade de Medicina \\ Universidade de São Paulo, \\ São Paulo, Brasil. \\ Correspondência \\ M. Andreotti \\ Sub-gerência de Vigilância \\ em Saúde do Trabalhador, \\ Coordenadoria de Vigilância \\ em Saúde, Secretaria \\ Municipal de Saúde. \\ Rua Santa Isabel 181, \\ São Paulo, SP \\ 01221-010, Brasil.
}

\begin{abstract}
To estimate the risk of occupation in oral and oropharyngeal cancer, a hospital-based casecontrol study was conducted in Greater Metropolitan São Paulo, Brazil. The study included 325 cases and 468 controls, frequency-matched with cases by sex and age, and interviewed from January 1999 to March 2002. Occupational risks were examined by industry and job titles and restricted to males (266 cases, 362 controls). Odds ratios (OR) were calculated by unconditional logistic regression, controlling for age, smoking, and alcohol. Males working in vehicle maintenance shops (26 cases, 12 controls) showed adjusted $O R=2.45$ (95\%CI: 1.14-5.27), increasing among those employed 10 or more years $(\mathrm{OR}=$ 7.90; 95\%CI: 2.03-30.72). Occupation as vehicle repair worker (14 cases, 7 controls) showed $O R=$ 2.10 (95\%CI: 0.78-5.68), increasing among workers exposed 10 or more years $(O R=26.21 ; 95 \% C I$ : 2.34-294.06). Other industries and occupations revealed OR higher than 1.5, but not statistically significant. In conclusion, employment in vehicle maintenance shops and occupation as vehicle repair worker showed risk for oral cancer, independently of smoking and alcohol. More prolonged exposure increased the risk.
\end{abstract}

Mouth Neoplasms; Oropharyngeal Neoplasms; Occupational Diseases

\author{
Magda Andreotti 1,2 \\ Aparecida Natália Rodrigues ${ }^{2}$ \\ Luiza Maria Nunes Cardoso ${ }^{3}$ \\ Rejane A. de Oliveira Figueiredo 2 \\ José Eluf-Neto 4 \\ Victor Wünsch-Filho ${ }^{2}$
}

\section{Introdução}

Os tumores de cabeça e pescoço pela expressiva incidência e mortalidade, assim como alta letalidade, constituem-se em relevante preocupação para a saúde no mundo, particularmente nos países em desenvolvimento ${ }^{1}$. No Brasil, de acordo com recentes estimativas, anualmente são registrados cerca de 13 mil casos novos e mais de 3 mil mortes pela doença (http://www. inca.gov.br/estimativa/2005, acessado em 28/ Dez/2004). É a mais alta incidência na América Latina, mas com grandes variações por regiões geográficas do país 2 . A Cidade de São Paulo registra isoladamente a mais alta incidência na área das Américas ${ }^{3}$. As tendências temporais nas cidades de São Paulo 4,5 e Goiânia 6,7,8 revelam incidências crescentes, inferindo-se que o mesmo esteja ocorrendo no país como um todo. O diagnóstico do câncer oral no Brasil é, em geral, realizado mais tardiamente do que em países desenvolvidos 9 .

Fumo e álcool são os principais fatores de risco reconhecidos, mas estudos prévios encontraram relação entre câncer da cavidade bucal e faringe e o exercício de determinadas ocupações, como por exemplo: pescadores e agricultores 10 , pintores 11,12 , açougueiros, pedreiros e condutores de veículos a motor 13, encanadores e trabalhadores da construção civil 14, instaladores de carpetes 15. Exposições ocupacionais a substâncias químicas específicas tam- 
bém têm sido apontadas como relacionadas às neoplasias de cavidade oral e faringe, tais como formaldeído ${ }^{14}$, fenoxi herbicidas e dioxinas 16 . No Brasil não há pesquisas reportando especificamente os efeitos da ocupação na gênese do câncer da cavidade bucal e orofaringe.

O objetivo neste estudo foi investigar o papel dos fatores ocupacionais, avaliados por meio do emprego em distintos ramos de atividade e da ocupação exercida, na incidência do câncer da cavidade oral e orofaringe na Região Metropolitana de São Paulo.

\section{Métodos}

O presente estudo caso-controle de base hospitalar é parte de um estudo multicêntrico internacional conduzido na América Latina e coordenado pela Agência Internacional de Pesquisas em Câncer (International Agency for Research on Cancer - IARC) da Organização Mundial da Saúde. A área do estudo, a Região Metropolitana de São Paulo, compreende $37 \mathrm{mu}$ nicípios e cerca de 18,8 milhões de habitantes. A força de trabalho inclui cerca de 9,4 milhões de habitantes, dos quais $20,3 \%$ trabalham na indústria, $2,7 \%$ na construção civil, $16,1 \%$ no comércio, $52,3 \%$ no setor de serviços e $8,6 \%$ como empregados domésticos (http://www.seade.gov.br/produtos/anuário/index.htm, acessado em 28/Dez/2004).

Entre primeiro de janeiro de 1999 e 31 de dezembro de 2001, os casos do estudo foram rastreados e entrevistados em sete hospitais da Cidade de São Paulo que concentram a atenção médica a pacientes com câncer da cavidade bucal e orofaringe, oriundos da Região Metropolitana de São Paulo. Os pacientes, residentes na região por no mínimo seis meses, com diagnósticos recentes e confirmados por histologia, e que não haviam sido submetidos a tratamento prévio para a doença foram incluídos no estudo.

O recrutamento de controles realizou-se entre 1o de fevereiro de 1999 e 31 de março de 2002 nos mesmos hospitais de origem dos casos. Também eram residentes na Região Metropolitana de São Paulo por no mínimo seis meses. Os controles foram recrutados em relação aos casos por freqüência em estratos de faixa etária, por qüinqüênios e por sexo. Os diagnósticos dos controles foram obtidos dos prontuários médicos e codificados de acordo com a Classificação Internacional de Doenças - 10a Revisão (CID-10) 17. Indivíduos com história clínica atual ou pregressa, suspeita de neoplasia da cavidade oral e orofaringe, foram excluídos, bem como pacientes com doenças asso- ciadas aos fatores de risco sob investigação neste estudo. Excluíram-se pacientes com graves impedimentos clínicos para responder o questionário.

O estudo foi aprovado pelos comitês de ética dos hospitais onde foi conduzido e, também, pela Comissão Nacional de Ética em Pesquisa. O consentimento por escrito para participação no estudo foi obtido de casos e controles.

Foram identificados inicialmente para entrevista 1.037 indivíduos, 509 casos e 528 controles. Desses casos, 35 não foram confirmados, 130 referiram residir menos de seis meses na Região Metropolitana de São Paulo e 14 recusaram-se a participar da pesquisa. Dos controles, 45 residiam menos de seis meses na Região Metropolitana de São Paulo e 15 não aceitaram participar.

Casos e controles que preenchiam os critérios de inclusão eram abordados no hospital, no atendimento ambulatorial ou na internação. A maioria dos casos foi entrevistada durante os procedimentos da investigação clínica para confirmação diagnóstica. As entrevistas foram realizadas por uma equipe de entrevistadores treinada especificamente para aplicar o questionário desenvolvido para o estudo.

\section{Coleta de dados}

O instrumento para coleta de dados compunhase de três partes: questionário sobre hábitos de vida (QHV), questionário ocupacional geral (QOG) e questionários ocupacionais especializados (QOE). O QHV comportava questões sobre saúde, padrões socioculturais e vários fatores de risco, como dieta, consumo de tabaco e álcool, e ocorrência de câncer em familiares.

A variável tabagismo foi mensurada considerando-se a experiência de fumante de cada indivíduo. Essa experiência foi definida em termos de exposição cumulativa considerando-se o número de maços de cigarros consumidos por dia e o tempo de tabagismo. Tanto o consumo de cigarros quanto de charutos e cachimbo foi estimado: um charuto correspondendo a quatro cigarros e um fornilho de cachimbo a três cigarros 18. A média diária de consumo de maços (com vinte cigarros) foi calculada e multiplicada pelo número de anos que o indivíduo foi fumante (maços-ano).

Para a ingestão de álcool, o consumo médio diário foi considerado em gramas. Então, o consumo de bebidas referido pelo indivíduo foi transformado em gramas de álcool. Foram utilizados os seguintes critérios de conversão para gramas de álcool por litro: cerveja, $40 \mathrm{~g}$; vinho, 96g; cachaça, 328g; e licores, 240g 19. 
O levantamento da história ocupacional do indivíduo, por meio do QOG, relacionou os empregos, em ordem cronológica, aos quais o entrevistado esteve vinculado durante a vida até o momento da entrevista. Foram obtidas informações sobre a idade do indivíduo ao início e término do exercício da ocupação, o cargo que ocupou, bem como a razão social, o respectivo endereço, cidade e ramo de atividade ou setor produtivo da empresa ou do empregador. Foram investigadas e registradas as lacunas de tempo entre um emprego e outro, buscando-se identificar períodos de desemprego, adoecimento, trabalho domiciliar ou a sobreposição de tempo de serviço por justaposição de vínculos de trabalho e períodos de trabalhos sazonais ou temporários. Mudanças significativas de função ou processo produtivo em uma mesma empresa foram registrados separadamente.

\section{Codificação das variáveis ramo de atividade e ocupação}

A Classificação Estatística para Atividades Econômicas na Comunidade Européia (Statistical Classification of Economic Activities in the European Community - NACE (http://unstats.un. org/unsd/class/intercop/training/australia/pa pers/austra-15.pps, acessado em 28/Dez/2004) foi utilizada para classificar ramo de atividade. Essa classificação apresenta correspondência com a Classificação Padrão para Classificação Industrial de Todas as Atividades Econômicas (The International Standard Industrial Classification of All Economic Activities - ISIC (http:// www.ilo.org/public/english/bureau/stat/class/ isic.htm, acessado em 28/Dez/2004) e, também, com a Classificação Nacional de Atividades Econômicas (http://www.ibge.gov.br/cgi-bin/cnaeprd.html, acessado em 28/Dez/2004). A atividade principal da empresa onde o entrevistado trabalhou foi codificada considerando-se o processo produtivo e a produção de bens e serviços.

Para a classificação da ocupação, utilizouse a Classificação Internacional para Ocupações (International Standard Classification of Occupations - ISCO) da Organização Internacional do Trabalho (http://www.ilo.org/public/ english/bureau/stat/isco/index.htm, acessado em 28/Dez/2004), que é a base da Classificação Brasileira de Ocupações (http://www.mte.gov. br/empregador/caged/estatistica/nocupacional/default.asp, acessado em 28/Dez/2004). Para cada participante do estudo foram verificadas as informações obtidas nos questionários sobre a profissão: tipo de empresa, matéria-prima empregada, o produto final, ferramentas utilizadas, o tempo exigido para executar cada atividade principal e atividades complementares. A caracterização das tarefas executadas permitiu incluir a ocupação no código mais representativo dentre as categorias específicas em cada agrupamento profissional.

Foram considerados válidos para a codificação os empregos com período igual ou maior que seis meses. Trabalhos fora do mercado formal de trabalho também foram avaliados para fins de inclusão ou não no perfil ocupacional e posterior análise como exposição aos fatores de risco. Para a codificação dos períodos caracterizados como não-ocupacionais, tais como serviço militar obrigatório, aposentadoria, desemprego, serviço doméstico, adoecimento e reclusão foram criados códigos especiais, bem como para as ocupações que não puderam ser identificadas.

\section{Análise estatística}

A análise dos riscos ocupacionais foi possível apenas para os homens (266 casos e 362 controles com informações válidas para as variáveis ocupacionais), em decorrência do pequeno número de mulheres com tumores orais e faringe $(n=51)$ e sua dispersão por muitos ramos de atividade e ocupações. Selecionou-se para análise apenas os ramos de atividade e as ocupações que comportavam, no mínimo, cinco indivíduos (casos ou controles).

O risco de câncer da cavidade bucal e orofaringe foi estimado comparando-se indivíduos que estiveram alguma vez empregados em um ramo de atividade ou categoria ocupacional com aqueles que nunca trabalharam naquele segmento econômico ou ocupação. Portanto, um mesmo indivíduo pode ter sido incluído em mais de um grupo de atividade econômica ou ocupação, de acordo com sua trajetória ocupacional.

Tanto para as atividades econômicas quanto para as ocupações, a duração da exposição caracterizada pelo tempo de permanência no emprego foi agrupada em duas classes: alguma vez empregado por qualquer período de tempo e, outra, de dez ou mais anos no emprego ou exercício da profissão. Para a análise de latência, período compreendido entre o início da exposição (primeiro emprego em determinado ramo de atividade ou início na ocupação) e o diagnóstico clínico do câncer, considerou-se o período mínimo de vinte anos.

Odds ratios (OR) e respectivos intervalos com 95\% de confiança (IC95\%) foram calculados como aproximações do risco relativo. Potenciais variáveis de confusão - idade, consumo de tabaco e álcool - foram controladas no modelo de regressão logística não condicional 20,21. A idade, variável de emparelhamento por 
freqüência, foi incluída no modelo de regressão logística, pois as variáveis de emparelhamento embora otimizem a busca de controles, nos estudos caso-controle mantém o seu potencial efeito de confundimento 21 .

Nos procedimentos de análise utilizaramse os programas estatísticos SAS ${ }^{\circledR}$ (SAS Institute, Cary, Estados Unidos) e SPSS 10.0 for Windows ${ }^{\circledR}$ (SPSS Inc., Chicago, Estados Unidos).

\section{Resultados}

A relação entre casos e controles foi 1:1,3 entre os homens e 1:1,9 entre as mulheres. A média de idade dos casos foi de 55 anos e dos controles 56,3. A maioria dos casos e controles foi composta por indivíduos da cor branca. Não houve diferença estatisticamente significante entre casos e controles em relação ao nível educacional. A rotatividade de empregos durante a vida, nos indivíduos do sexo masculino, foi semelhante nos casos (média de 2,9 empregos) e controles (média de 3,1 empregos). Nas mulheres, a média de empregos entre os casos foi de 2,6 empregos e nos controles 2,2 empregos. As características sócio-demográficas dos 325 casos e 468 controles estão descritas na Tabela 1.

Tabela 1

Distribuição de casos e controles de acordo com variáveis sócio-demográficas.

\begin{tabular}{|c|c|c|c|c|}
\hline \multirow[t]{2}{*}{ Variáveis } & \multicolumn{2}{|c|}{ Casos $(n=325)$} & \multicolumn{2}{|c|}{ Controles $(n=468)$} \\
\hline & $n$ & $\%$ & $n$ & $\%$ \\
\hline \multicolumn{5}{|l|}{ Sexo } \\
\hline Masculino & 274 & 84,6 & 369 & 81,1 \\
\hline Feminino & 51 & 15,4 & 99 & 18,9 \\
\hline \multicolumn{5}{|l|}{ Idade (anos)* } \\
\hline$<50$ & 103 & 31,7 & 142 & 30,3 \\
\hline $50-59$ & 114 & 35,1 & 134 & 28,6 \\
\hline $60-69$ & 73 & 22,5 & 116 & 24,8 \\
\hline$\geq 70$ & 35 & 10,8 & 76 & 16,2 \\
\hline \multicolumn{5}{|l|}{ Cor** } \\
\hline Branca & 222 & 68,3 & 318 & 68,0 \\
\hline Não-branca & 92 & 31,7 & 143 & 32,0 \\
\hline \multicolumn{5}{|l|}{ Escolaridade $e^{\star \star \star}$} \\
\hline Analfabeto & 44 & 13,5 & 65 & 13,9 \\
\hline Fundamental & 234 & 72,0 & 319 & 68,2 \\
\hline Médio/Técnico & 38 & 11,7 & 56 & 12,0 \\
\hline Superior & 9 & 2,8 & 27 & 5,8 \\
\hline
\end{tabular}

* Idade média dos casos 55 anos (desvio padrão = 10,9 anos); controles 56,3 anos (desvio padrão $=12,3$ anos).

** Informação não disponível para 11 casos e 7 controles.

*** Informação não disponível para um controle.
A localização anatômica mais freqüente dos tumores da cavidade bucal e orofaringe, incluídos neste estudo, tanto nos indivíduos do sexo masculino quanto do feminino, foi a língua (Tabela 2).

Fumantes apresentaram risco elevado quando comparados com os indivíduos que nunca fumaram. Evidenciou-se efeito dose-resposta, com riscos crescentes na relação direta do consumo em maços-ano. Também os consumidores de bebidas alcoólicas, comparados aos indivíduos que nunca beberam, apresentaram risco. O alto consumo de bebidas alcoólicas (níveis de etanol acima de $300 \mathrm{~g} /$ litro/dia) emergiu isoladamente como o fator de risco associado de forma mais intensa ao câncer da cavidade bucal e faringe (Tabela 3 ).

O emprego em oficinas mecânicas revelou risco de câncer da cavidade bucal e orofaringe (OR = 2,45; IC95\%: 1,14-5,27), que aumentou naqueles com dez ou mais anos de emprego nesta atividade econômica $(\mathrm{OR}=7,90$; IC95\%: 2,03-30,72). A adição na análise do período de latência (vinte ou mais anos de início do exercício na atividade) não modificou o risco observado para aqueles expostos por dez ou mais anos (Tabela 4).

Os setores de mineração, manufatura de artigos de madeira, indústria de papel e papelão, indústria química, setores do comércio atacadista e varejista, de hotelaria e restaurante, de intermediação financeira, da educação e de serviços de saúde e sociais, particularmente para aqueles com longa exposição, também apresentaram excesso de risco, porém mais discretos e não estatisticamente significativos (Tabela 4).

Os indivíduos que exerceram a ocupação de mecânicos de veículos apresentaram risco elevado, porém sem significância estatística $(\mathrm{OR}=$ 2,10; IC95\%: 0,78-5,68). O exercício dessa ocupação por dez ou mais anos multiplicou o risco, que se tornou estatisticamente significante (OR = 26,21; IC95\%: 2,34-294,06) (Tabela 5).

Outras categorias ocupacionais, como administradores e gerentes, vendedores e balconistas, cozinheiros e garçons, trabalhadores da indústria de alimentos e gráficos, em situações de longa exposição, também revelaram risco para o câncer da boca e faringe, mas sem significância estatística (Tabela 5).

\section{Discussão}

A abordagem utilizada neste estudo para avaliar o efeito dos fatores ocupacionais, por ramo de atividade e ocupação, não permite inferên- 
cias sobre exposições específicas, pois foi elaborada sem a construção de hipóteses $a$ priori sobre os agentes físicos e químicos ocupacionais possivelmente associados aos tumores da cavidade bucal e orofaringe. A principal evidência constatada, de que o emprego em oficinas mecânicas e a ocupação de mecânico de automóveis representam risco para câncer da cavidade bucal e orofaringe é inusitada, pois não há referências sobre tal associação em estudos publicados em outros países. De fato, Vaughan et al. 22, nos Estados Unidos, identificaram riscos elevados de câncer da faringe e cavidade sinusal para mecânicos industriais e mecânicos de veículos, porém sem significância estatística.

Mecânicos de veículos estão expostos habitualmente a vapores derivados da combustão de motores a gasolina, diesel ou álcool anidro; solventes; névoas de óleos minerais lubrificantes e ácidos fortes; partículas de materiais isolantes como fibras de amianto e de vidro; poeiras metálicas e abrasivas; aldeídos; fumos de solda e fuligem; entre outros. Esses agentes químicos foram revisados nas monografias da IARC 23 e a maioria foi classificada como definitivamente carcinogênica para os humanos (Grupo 1), outra parte como provavelmente carcinogênica (Grupo 2A) e uma pequena parcela como possivelmente carcinogênica (Grupo 2B). Isto confere plausibilidade biológica para $\mathrm{o}$ achado mais relevante deste estudo. $\mathrm{O}$ fato de o risco ter sido detectado tanto para a atividade em oficinas mecânicas como para a ocupação de mecânico de veículos, bem como o aumento do risco nos expostos por dez ou mais anos, estabelece consistência aos resultados encontrados.

Outras atividades econômicas, como mineração, industrialização de artigos de madeira, indústria de papel e papelão, indústria química, comércio atacadista e varejista, intermediação financeira, educação e serviços de saúde, também apresentaram risco para câncer da cavidade bucal e faringe; em geral, tênues e não estatisticamente significativos. São resultados compatíveis com estudos que identificaram risco em algumas dessas atividades, embora outras pesquisas não tenham apontado tais riscos. Discutem-se, a seguir, algumas dessas evidências com relação ao observado em estudos realizados em outros países.

Na manufatura de artigos de madeira alguns autores observaram risco de tumores da boca para os expostos por longo tempo, porém sem significância estatística 12,14. Tais resultados são semelhantes ao obtido neste estudo. As substâncias carcinogênicas reconhecidas nesse setor são: poeira de madeira e seus conser-
Tabela 2

Localização anatômica dos tumores incluídos no estudo.

\begin{tabular}{lrrrrr}
\hline \multirow{2}{*}{ Localização (CID-10) } & \multicolumn{2}{c}{ Homens } & \multicolumn{2}{c}{ Mulheres } \\
& $\mathrm{n}$ & $\%$ & $\mathrm{n}$ & $\%$ \\
\hline Língua (C01-C02) & 96 & 35,0 & 22 & 43,1 \\
Gengiva (C03) & 9 & 3,3 & 3 & 5,9 \\
Assoalho da boca (C04) & 36 & 13,1 & 5 & 9,8 \\
Palato (C05) & 19 & 7,0 & 5 & 9,8 \\
Boca (C06) & 35 & 12,8 & 9 & 17,7 \\
Amígdalas (C09) & 45 & 15,7 & 2 & 3,9 \\
Orofaringe (C10) & 33 & 12,0 & 5 & 9,8 \\
Localizações mal definidas (C14) & 3 & 1,1 & 0 & 0,0 \\
Total & 274 & 100,0 & 51 & 100,0 \\
\hline
\end{tabular}

CID-10 = Classificação Estatística Internacional de Doenças

e Problemas Relacionados à Saúde 17.

Tabela 3

Análise univariada do risco de tabagismo e consumo de bebida alcoólica no câncer bucal e orofaringe.

\begin{tabular}{|c|c|c|c|c|}
\hline Variáveis & Casos & Controles & OR bruta & IC95\% \\
\hline \multicolumn{5}{|l|}{ Tabagismo } \\
\hline Nunca fumou & 16 & 134 & 1,0 & \\
\hline Ex-fumante* & 56 & 160 & 2,9 & $1,6-5,3$ \\
\hline Fumante atual & 252 & 173 & 12,1 & $7,0-21,0$ \\
\hline \multicolumn{5}{|l|}{ Tabagismo (maços-ano) ${ }^{\star \star}$} \\
\hline Nunca fumou & 18 & 135 & 1,0 & \\
\hline$\leq 20$ & 64 & 108 & 4,4 & $2,5-7,9$ \\
\hline $21-39$ & 118 & 117 & 7,6 & $4,3-13,2$ \\
\hline$\geq 40$ & 117 & 94 & 9,3 & $5,3-16,4$ \\
\hline \multicolumn{5}{|l|}{ Bebidas alcoólicas } \\
\hline Nunca bebeu & 21 & 140 & 1,0 & \\
\hline Bebeu no passado*** & 132 & 180 & 4,9 & $2,9-8,1$ \\
\hline Bebe atualmente & 170 & 147 & 7,7 & $4,6-12,8$ \\
\hline \multicolumn{5}{|c|}{ Consumo de etanol (g/L/dia)\# } \\
\hline Nunca bebeu & 21 & 140 & 1,0 & \\
\hline$\leq 100$ & 179 & 242 & 4,9 & $3,0-8,1$ \\
\hline $100-300$ & 96 & 69 & 9,3 & $5,3-16,1$ \\
\hline$>300$ & 24 & 6 & 26,7 & $9,8-72,9$ \\
\hline
\end{tabular}

OR bruta = odds ratio bruta; IC95\% = intervalo com 95\% de confiança.

* Indivíduos que cessaram de fumar há mais de um ano da data da entrevista.

** Consumo em maços-ano: informação não disponível para 9 casos e 14 controles.

*** Indivíduos que cessaram o consumo de bebidas alcoólicas há mais de um ano da data da entrevista.

\# Consumo de etanol em gramas/litro/dias: informação não disponível para 5 casos e 11 controles. 
Emprego em atividades econômicas selecionadas e risco de câncer da cavidade bucal e orofaringe. Homens.

\begin{tabular}{|c|c|c|c|c|c|c|c|c|c|c|c|c|}
\hline \multirow[t]{2}{*}{ Atividade econômica*, } & \multicolumn{4}{|c|}{ Empregados ${ }^{\star \star \star}$} & \multicolumn{4}{|c|}{ Empregados $\geq 10$ anos ${ }^{\star \star \star}$} & \multicolumn{4}{|c|}{$\begin{array}{c}\text { Empregados } \geq 10 \text { anos } \\
\text { e } \geq 20 \text { anos de latência }\end{array}$} \\
\hline & $\begin{array}{c}\text { Casos } \\
(n=262)\end{array}$ & $\begin{array}{l}\text { Controles } \\
(n=345)\end{array}$ & OR & IC95\% & $\begin{array}{c}\text { Casos } \\
(n=233)\end{array}$ & $\begin{array}{r}\text { Controles } \\
(n=305)\end{array}$ & OR & IC95\% & $\begin{array}{c}\text { Casos } \\
(n=224)\end{array}$ & $\begin{array}{l}\text { Controles } \\
(n=277)\end{array}$ & OR & IC95\% \\
\hline Agricultura (011-3) & 84 & 147 & 0,68 & $0,47-0,98$ & 46 & 85 & 0,66 & $0,41-1,04$ & 46 & 85 & 0,66 & $0,41-1,04$ \\
\hline Mineração (131-2) & 7 & 8 & 0,95 & $0,32-2,86$ & 3 & 3 & 1,73 & $0,26-11,49$ & 3 & 3 & 1,73 & $0,26-11,49$ \\
\hline Alimentos e bebidas (15) & 28 & 36 & 1,13 & $0,63-2,01$ & 4 & 13 & 0,46 & $0,13-1,58$ & 4 & 12 & 0,49 & $0,14-1,76$ \\
\hline Têxtil (17-18) & 23 & 21 & 1,64 & $0,83-3,24$ & 8 & 9 & 1,14 & $0,40-3,28$ & 7 & 8 & 1,12 & $0,37-3,39$ \\
\hline $\begin{array}{l}\text { Fábrica de artigos } \\
\text { de madeira (20) }\end{array}$ & 6 & 6 & 1,40 & $0,40-4,94$ & 2 & 1 & 2,25 & $0,20-25,65$ & 2 & 1 & 2,25 & $0,20-25,65$ \\
\hline Papel e papelão (21) & 10 & 18 & 0,69 & $0,30-1,62$ & 7 & 5 & 1,32 & $0,37-4,75$ & 7 & 3 & 1,80 & $0,42-7,70$ \\
\hline Química (24) & 21 & 18 & 1,57 & $0,76-3,23$ & 5 & 2 & 4,20 & $0,58-30,17$ & 5 & 2 & 4,20 & $0,58-30,17$ \\
\hline Borracha e plástico (25) & 9 & 24 & 0,46 & $0,20-1,07$ & 2 & 6 & 0,51 & $0,09-2,74$ & 2 & 6 & 0,51 & $0,09-2,74$ \\
\hline Vidreira e cerâmica (261-8) & 20 & 18 & 1,43 & $0,69-2,95$ & 8 & 8 & 1,30 & $0,43-3,96$ & 7 & 8 & 1,11 & $0,35-3,53$ \\
\hline Metalurgia básica (27-29) & 55 & 78 & 0,85 & $0,55-1,30$ & 20 & 34 & 0,80 & $0,42-1,51$ & 18 & 31 & 0,73 & $0,38-1,43$ \\
\hline $\begin{array}{l}\text { Fábrica de equipamentos } \\
\text { elétricos ( } 30)\end{array}$ & 13 & 19 & 0,79 & $0,36-1,72$ & 5 & 7 & 0,89 & $0,25-3,17$ & 4 & 6 & 0,85 & $0,21-3,44$ \\
\hline $\begin{array}{l}\text { Fábrica de equipamentos } \\
\text { de transporte (34-35) }\end{array}$ & 17 & 40 & 0,44 & $0,23-0,83$ & 8 & 15 & 0,69 & $0,26-1,83$ & 8 & 14 & 0,69 & $0,26-1,83$ \\
\hline $\begin{array}{l}\text { Fábrica de artigos } \\
\text { mobiliário (361) }\end{array}$ & 17 & 29 & 0,67 & $0,34-1,30$ & 7 & 11 & 0,66 & $0,22-1,96$ & 6 & 10 & 0,65 & $0,20-2,11$ \\
\hline Construção civil (451-5) & 87 & 97 & 1,17 & $0,80-1,70$ & 56 & 63 & 1,13 & $0,72-1,76$ & 48 & 48 & 1,25 & $0,76-2,03$ \\
\hline $\begin{array}{l}\text { Comércio atacadista } \\
\text { e varejista }(50-52)\end{array}$ & 83 & 105 & 1,20 & $0,82-1,75$ & 47 & 56 & 1,42 & $0,87-2,31$ & 42 & 44 & 1,59 & $0,94-2,68$ \\
\hline Oficina mecânica (502) & 26 & 12 & 2,45 & $1,14-5,27$ & 21 & 3 & 7,90 & $2,03-30,72$ & 19 & 3 & 7,38 & $1,88-28,98$ \\
\hline Hotelaria e restaurante (55) & 28 & 29 & 1,50 & $0,82-2,74$ & 12 & 16 & 1,30 & $0,57-2,98$ & 9 & 11 & 1,50 & $0,57-3,95$ \\
\hline $\begin{array}{l}\text { Transporte e comunicação } \\
(60-62)\end{array}$ & 52 & 80 & 0,82 & $0,53-1,26$ & 26 & 44 & 0,78 & $0,44-1,39$ & 21 & 38 & 0,78 & $0,42-1,46$ \\
\hline Intermediação financeira (65) & 10 & 17 & 0,92 & $0,39-2,20$ & 5 & 4 & 2,05 & $0,47-9,07$ & 5 & 4 & 2,05 & $0,47-9,07$ \\
\hline $\begin{array}{l}\text { Imobiliário, prestação } \\
\text { de serviço (70-73) }\end{array}$ & 20 & 51 & 0,43 & $0,24-0,78$ & 9 & 23 & 0,44 & $0,18-1,06$ & 7 & 18 & 0,43 & $0,16-1,20$ \\
\hline Administração pública (75) & 19 & 41 & 0,64 & $0,35-1,16$ & 10 & 14 & 0,93 & $0,37-2,30$ & 8 & 11 & 0,88 & $0,32-2,43$ \\
\hline Educação (80) & 8 & 13 & 1,11 & $0,42-2,96$ & 2 & 3 & 1,52 & $0,24-9,53$ & 2 & 2 & 2,11 & $0,28-15,72$ \\
\hline Serviços de saúde, sociais (85) & 10 & 9 & 1,44 & $0,53-3,91$ & 6 & 3 & 2,15 & $0,46-9,95$ & 5 & 1 & 6,00 & $0,65-55,34$ \\
\hline Serviços pessoais e col. (90-99) & 23 & 47 & 0,57 & $0,32-1,01$ & 7 & 28 & 0,28 & $0,11-0,69$ & 4 & 22 & 0,20 & $0,06-0,64$ \\
\hline
\end{tabular}

* Categorias com no mínimo cinco indivíduos empregados.

** Entre parênteses códigos da Statistical Classification of Economic Activities in the European Community (NACE)

*** Categoria de referência: nunca empregados na atividade sob avaliação.

$\mathrm{OR}=$ odds ratio ajustada por idade, tabagismo e consumo de bebida alcoólica; IC $95 \%$ = intervalo com $95 \%$ de confiança.

vantes, formaldeído e solventes presentes em colas, vernizes, lacas, selantes e resinas.

$\mathrm{Na}$ indústria química o risco encontrado foi mais intenso nos empregados nesse segmento industrial por dez ou mais anos. Resultado que apresenta correspondência com o obtido por Merletti et al. 14, que identificaram risco estatisticamente significativo de câncer da cavidade oral naquele ramo de atividade. Huebner et al. 15 não chegaram às mesmas conclusões, porém, ao discriminarem os riscos por localização anatômica do tumor, encontraram risco para câncer de língua em trabalhadores de refinarias de petróleo.
O trabalho exercido no segmento do comércio atacadista e varejista apresentou aumento de risco sem significância estatística, resultado semelhante para as ocupações de vendedores e balconistas. É um resultado compatível com os publicados por Pukkala et al. 10 e Huebner et al. 15, mas difere do obtido por Merletti et al. 14 .

O risco apontado na área da educação apresenta concordância de direção com o resultado encontrado por Pukkala et al. 10, que destacaram particularmente a ocupação de professor do sexo feminino com risco elevado para o câncer oral. No ramo de serviços de saúde e sociais o risco mostrou-se crescente neste estu- 
Ocupações selecionadas e risco de câncer da cavidade bucal e orofaringe. Homens.

\begin{tabular}{|c|c|c|c|c|c|c|c|c|c|c|c|c|}
\hline \multirow[t]{2}{*}{ Ocupação*,** } & \multicolumn{4}{|c|}{ Empregados ${ }^{\star \star \star}$} & \multicolumn{4}{|c|}{ Empregados $\geq 10$ anos $^{\star \star \star}$} & \multicolumn{4}{|c|}{$\begin{array}{c}\text { Empregados } \geq 10 \text { anos } \\
\text { e } \geq 20 \text { anos de latência }{ }^{\star \star \star}\end{array}$} \\
\hline & $\begin{array}{c}\text { Casos } \\
(n=262)\end{array}$ & $\begin{array}{l}\text { Controles } \\
(n=344)\end{array}$ & OR & IC95\% & $\begin{array}{c}\text { Casos } \\
(n=231)\end{array}$ & $\begin{array}{l}\text { Controles } \\
(n=304)\end{array}$ & OR & $1 \mathrm{C} 95 \%$ & $\begin{array}{l}\text { Casos } \\
(\mathrm{n}=219)\end{array}$ & $\begin{array}{r}\text { Controles } \\
(n=280)\end{array}$ & OR & IC95\% \\
\hline $\begin{array}{l}\text { Profissionais e técnicos } \\
(01-19)\end{array}$ & 19 & 37 & 0,78 & $0,41-1,48$ & 12 & 21 & 0,90 & $0,41-2,01$ & 12 & 21 & 0,90 & $0,41-2,01$ \\
\hline $\begin{array}{l}\text { Administradores } \\
\text { e gerentes }(20)\end{array}$ & 13 & 17 & 1,15 & $0,51-2,60$ & 5 & 7 & 1,20 & $0,32-4,46$ & 5 & 5 & 1,83 & $0,42-7,96$ \\
\hline Escriturário e assemel.(30-39) & 47 & 72 & 0,84 & $0,54-1,31$ & 22 & 32 & 0,81 & $0,43-1,53$ & 22 & 31 & 0,84 & $0,44-1,58$ \\
\hline Cobrador de ônibus (36) & 7 & 13 & 0,74 & $0,27-2,02$ & 0 & 3 & - & - & 0 & 3 & - & - \\
\hline Vendedor, balconista (40-49) & 52 & 75 & 1,14 & $0,74-1,77$ & 33 & 39 & 1,40 & $0,80-2,47$ & 26 & 28 & 1,60 & $0,85-3,02$ \\
\hline $\begin{array}{l}\text { Faxineiro, diarista, cabeleireiro } \\
(50-59)\end{array}$ & 33 & 70 & 0,59 & $0,37-0,96$ & 17 & 45 & 0,52 & $0,28-0,98$ & 13 & 30 & 0,61 & $0,29-1,28$ \\
\hline Cozinheiro, garçons (531-2) & 19 & 20 & 1,47 & $0,73-2,98$ & 9 & 11 & 1,76 & $0,65-4,79$ & 6 & 9 & 1,69 & $0,52-5,47$ \\
\hline Agricultores (62) & 84 & 152 & 0,62 & $0,43-0,89$ & 50 & 87 & 0,65 & $0,41-1,02$ & 50 & 86 & 0,66 & $0,42-1,04$ \\
\hline Trabalhador de fundição (72) & 10 & 17 & 0,73 & $0,31-1,73$ & 1 & 6 & 0,28 & $0,03-2,64$ & 1 & 5 & 0,30 & $0,03-2,86$ \\
\hline Têxteis (751-9) & 13 & 12 & 1,52 & $0,64-3,60$ & 3 & 5 & 0,73 & $0,17-3,15$ & 3 & 5 & 0,73 & $0,17-3,15$ \\
\hline $\begin{array}{l}\text { Trabalhador da indústria } \\
\text { de alimentos (77) }\end{array}$ & 13 & 14 & 1,06 & $0,45-2,48$ & 6 & 3 & 3,91 & $0,67-22,67$ & 6 & 3 & 3,91 & $0,67-22,67$ \\
\hline Marceneiros (81) & 14 & 19 & 0,96 & $0,45-2,06$ & 5 & 7 & 0,91 & $0,26-3,16$ & 5 & 6 & 1,14 & $0,31-4,18$ \\
\hline Metalúrgicos (83) & 29 & 39 & 0,91 & $0,52-1,58$ & 16 & 18 & 0,98 & $0,46-2,08$ & 15 & 17 & 0,93 & $0,43-2,02$ \\
\hline $\begin{array}{l}\text { Montador máquinas } \\
\text { e equipamentos ( } 84 \text { ) }\end{array}$ & 9 & 11 & 1,09 & $0,40-2,93$ & 6 & 8 & 1,21 & $0,34-4,24$ & 5 & 7 & 1,46 & $0,37-5,75$ \\
\hline Mecânico de veículos (843) & 14 & 7 & 2,10 & $0,78-5,68$ & 13 & 1 & 26,21 & $2,34-294,06$ & 11 & 1 & 24,46 & $2,10-284,60$ \\
\hline Eletricistas (85) & 14 & 15 & 1,08 & $0,48-2,41$ & 7 & 11 & 0,93 & $0,33-2,66$ & 6 & 8 & 1,08 & $0,34-3,41$ \\
\hline Soldadores, funileiros (87) & 18 & 25 & 0,71 & $0,35-1,42$ & 13 & 12 & 0,97 & $0,41-2,28$ & 13 & 12 & 0,97 & $0,41-2,28$ \\
\hline Vidreiros e ceramistas (89) & 8 & 13 & 0,84 & $0,31-2,25$ & 2 & 8 & 0,37 & $0,07-2,03$ & 2 & 8 & 0,37 & $0,07-2,03$ \\
\hline Gráficos (92) & 4 & 7 & 0,80 & $0,21-3,06$ & 4 & 2 & 1,73 & $0,24-12,51$ & 4 & 1 & 2,29 & $0,23-22,73$ \\
\hline Pintores (93) & 20 & 14 & 1,57 & $0,75-3,28$ & 13 & 12 & 1,13 & $0,48-2,69$ & 10 & 10 & 1,00 & $0,38-2,66$ \\
\hline Pedreiros (951) & 58 & 65 & 1,11 & $0,72-1,72$ & 37 & 41 & 1,12 & $0,66-1,92$ & 32 & 30 & 1,29 & $0,71-2,34$ \\
\hline $\begin{array}{l}\text { Operário de equipamentos } \\
\text { de carga (97) }\end{array}$ & 18 & 35 & 0,59 & $0,31-1,12$ & 5 & 13 & 0,39 & $0,12-1,19$ & 5 & 10 & 0,48 & $0,15-1,58$ \\
\hline Motorista de ônibus (98) & 47 & 51 & 1,29 & $0,80-2,09$ & 33 & 39 & 1,23 & $0,71-2,15$ & 26 & 34 & 1,17 & $0,64-2,14$ \\
\hline Ajudante geral (99) & 34 & 45 & 0,86 & $0,51-1,44$ & 6 & 13 & 0,73 & $0,25-2,10$ & 6 & 13 & 0,73 & $0,25-2,10$ \\
\hline
\end{tabular}

* Categorias com no mínimo cinco indivíduos empregados.

** Entre parênteses códigos da International Standard Classification of Occupations (ISCO).

$\star \star \star$ Categoria de referência: nunca exerceram a ocupação sob avaliação.

$\mathrm{OR}=$ odds ratio ajustada por idade, tabagismo e consumo de bebida alcoólica; IC $95 \%$ = intervalo com $95 \%$ de confiança

do, de acordo com o tempo de emprego e período de latência. Merletti et al. 14, identificaram risco para os trabalhadores do setor de serviços sociais, mas não para os de serviços de saúde, mesmo com longos períodos de exposição. Pukkala et al. 10 registraram riscos para o segmento de serviços em geral, incluídos os de saúde e sociais. Nos serviços de saúde o risco pode estar vinculado a exposições ao formaldeído e glutaraldeído (aldeídos), radiações ionizantes, quimioterápicos, óxido de etileno e alguns agentes biológicos.

O risco estimado para o trabalho na indústria de alimentos e ocupações correlatas (açougueiros, cozinheiros, garçons e barman) segue no geral os resultados referidos por outros estudos 13,14,24. Na indústria de alimentos esse risco pode ser atribuído à exposição aos hidrocarbonetos policíclicos aromáticos (HPA), decorrentes da combustão durante o cozimento de alimentos, bem como exposições a vírus oncogênicos, nitrosaminas, antioxidantes e fumos plásticos formados durante a termo-decomposição na embalagem de produtos alimentícios 25 . Tem sido assinalada a tendência ao consumo excessivo de cigarros e bebidas alcoólicas pelos indivíduos exercendo as ocupações de garçom e barman, mas neste estudo essas variáveis foram controladas na análise de regressão logística. 
Alguns aspectos da estrutura desta pesquisa podem ser assinalados como tendo influência nos resultados: falhas na obtenção da história ocupacional, erro de classificação não diferencial da exposição, e a influência de fatores de confusão não avaliados, como dieta, fatores genéticos, infecção por vírus. Análises que classificam os indivíduos pelo título da atividade econômica e da ocupação não permitem distinguir com precisão quais indivíduos foram ou não expostos a agentes carcinogênicos, e estão sujeitas a um potencial erro de classificação não diferencial, levando conseqüentemente à subestimação do risco 15. Assim, informações adicionais considerando-se exposições específicas a substâncias químicas ou agentes físicos são sempre necessárias para qualificar os riscos observados por este tipo de abordagem 26 .

A análise dos dados deste estudo restringiuse aos setores econômicos e ocupações com número mínimo de casos e controles (pelo menos cinco indivíduos, casos ou controles, em cada grupo). Portanto, não foi possível analisar algumas categorias reconhecidamente com exposições a substâncias cancerígenas. Isso limitou comparações mais amplas com os resultados obtidos por outros autores. Essas restrições impostas pela amostra disponível, certamente poderão ser superadas em análises futuras que incluam todo o conjunto de dados do estudo

\section{Resumo}

Estudo caso-controle conduzido para avaliar a associação de exposições ocupacionais e câncer oral e orofaringe na Região Metropolitana de São Paulo. Inclui 325 casos e 468 controles, recrutados por freqüencia de sexo e faixa etária, entrevistados entre janeiro de 1999 e março de 2002. A análise por ramos de atividade e ocupações foi restrita aos homens (266 casos, 362 controles) e os odds ratios (OR), calculados por regressão logística não-condicional, foram controlados por idade, tabagismo e consumo de álcool. Observou-se risco em trabalhadores de oficinas mecânicas (26 casos, 12 controles) $O R=2,45$ (IC95\%: 1,14-5,27), que aumentou nos que estavam empregados por dez ou mais anos $(O R=7,90$; IC95\%: 2,03-30,72). Os mecânicos de veícu- multicêntrico latino-americano, do qual os dados de São Paulo aqui apresentados fazem parte.

Em suma, os resultados deste estudo identificaram que a atividade em oficinas mecânicas e a ocupação como mecânico de veículos configuram-se como situações de risco para câncer da cavidade bucal e orofaringe, independentemente da idade e do consumo de tabaco e de álcool. Os riscos elevaram-se nas exposições prolongadas. Para avançar na análise dessas evidências será necessária a construção de matrizes de exposição ocupacional para detectar exposições específicas a agentes carcinogênicos 26 . Os resultados encontrados não apresentam correspondência com outros estudos realizados em outros países e, talvez, expressem aspectos singulares da estrutura socioeconômica brasileira. Diferente de outros países desenvolvidos, onde foram realizados estudos semelhantes, a frota automotiva mais antiga no Brasil exige reparos contínuos. Isso gera a multiplicação do número de oficinas mecânicas, particularmente nas periferias das grandes cidades brasileiras. Essas oficinas, em geral de pequeno porte, apresentam habitualmente precárias condições de higiene ocupacional. Os resultados assinalados indicam a necessidade de ações específicas de vigilância na área de saúde do trabalhador, para o controle da exposição a substâncias cancerígenas nesses ambientes. los (14 casos, 7 controles) apresentaram $O R=2,10$ (IC95\%: 0,78-5,68), e os expostos por 10 ou mais anos OR = 26,21 (IC95\%: 2,34-294,06). Outros ramos de atividade e ocupações apresentaram $O R \geq 1,5$, porém não estatisticamente significantes. Em conclusão, emprego em oficinas mecânicas e a profissão de mecânico de automóveis revelaram risco para câncer oral e orofaringe, independente da idade, tabaco e álcool. Longas exposições aumentaram o risco.

Neoplasias Bucais; Neoplasias Orofaríngeas; Doenças Ocupacionais 


\section{Colaboradores}

M. Andreotti foi responsável pela codificação dos ramos de atividade e das ocupações, pela análise dos dados e pela redação do artigo. A. N. Rodrigues participou na organização e gerenciamento do banco de dados da pesquisa. L. M. N. Cardoso colaborou na codificação dos ramos de atividade e das ocupações. R. A. O. Figueiredo contribuiu na supervisão da análise dos dados e na revisão do artigo. J. Eluf-Neto e V. Wünsch-Filho foram responsáveis pela coleta e análise dos dados e contribuíram na revisão crítica final do artigo.

\section{Agradecimentos}

Agradecemos a contribuição das equipes dos hospitais no centro de São Paulo integrantes do Estudo Multicêntrico Internacional na América Latina de Tumores de Cabeça e Pescoço: Dr. Luiz Paulo Kowalski, Hospital do Câncer A. C. Camargo; Dr. Marcos Brasilino de Carvalho, Hospital Heliópolis; Dr. José Francisco de Góis Filho, Instituto do Câncer Arnaldo Vieira de Carvalho; Dr. Antonio José Gonçalves, Santa Casa de Misericórdia de São Paulo; Dr. Márcio Abraão, Hospital São Paulo, Universidade Federal de São Paulo; equipes do Hospital do Servidor Público Estadual (IAMSPE) e do Hospital das Clínicas, Faculdade de Medicina, Universidade de São Paulo. Esta pesquisa teve o suporte financeiro da Comunidade Européia (IC18 CT970222) e da FAPESP (01/01768-2).

\section{Referências}

1. Pisani P, Bray F, Parkin DM. Estimates of the worldwide prevalence of cancer for 25 sites in the adult population. Int J Cancer 2002; 97:72-81.

2. Wünsch-Filho V. The epidemiology of oral and pharynx cancer in Brazil. Oral Oncol 2002; 38: 737-46.

3. Wünsch-Filho V, Camargo EA. The burden of mouth cancer in Latin America and the Caribbean: epidemiologic issues. Semin Oncol 2001; 28:158-68.

4. Mirra AP, organizador. Cancer incidence in São Paulo county, Brazil: 1983-1988-1993. Trend in the period 1969-1993. Brasília: Ministério da Saúde; 1999.

5. Mirra AP, Latorre MRDO, Veneziano DB, organizadores. Cancer incidence in the city of São Paulo: Brazil: 1997-1998. Cancer mortality in the city of São Paulo, Brazil: Trend in the period 19691998. Brasília: Ministério da Saúde; 2001.

6. Parkin DM, Muir CS, Whelan SL, Gao Y-T, Ferlay J, Powell J. Cancer incidence in five continents. v. VI. Lyon: International Agency for Research on Cancer; 1992. (IARC Scientific Publications, 120).

7. Parkin DM, Whelan SL, Ferlay J, Raymond L, Young J. Cancer incidence in five continents. v. VII. Lyon: International Agency for Research on Cancer; 1997. (IARC Scientific Publications, 143).

8. Parkin DM, Whelan SL, Ferlay J, Teppo L, Thomas DB. Cancer incidence in five continents. v. VIII. Lyon: International Agency for Research on Cancer; 2002. (IARC Scientific Publications, 155).

9. Carvalho AL, Singh B, Spiro RH, Kowalski LP, Shah JP. Cancer of the oral cavity: a comparison between institutions in a developing and a developed nation. Head Neck 2004; 26:31-8.

10. Pukkala E, Söderholm AL, Lindqvist C. Cancers of the lip and oropharynx in different social and occupational groups in Finland. Eur J Cancer B Oral Oncol 1994; 30B:209-15.

11. Skov T, Weiner J, Pukkala E, Malker H, Andersen A, Lynge E. Risk for cancer the pharynx and oral cavity among male painters in the Nordic countries. Arch Environ Health 1993; 48:176-80.

12. Brown LM, Moradi T, Gridley G, Plato N, Dosemeci M, Fraumeni-Junior JF. Exposures in the painting trades and paint manufacturing industry and risk among men and women in Sweden. J Occup Environ Med 2002; 44:258-64.

13. Orregia F, De Stefani E, Correa P, Rivero S, Fernán$\operatorname{dez}$ G, Leiva J, et al. Exposición ocupacional em el câncer oral, faríngeo e laríngeo. An Otorrinolaringol Ibero Am 1989; 16:365-76.

14. Merletti F, Boffetta P, Ferro G, Pisani P, Terracini B. Occupation and cancer of the oral cavity and oropharynx in Turin, Italy. Scand J Work Environ Health 1991; 17:248-54.

15. Huebner WW, Schoenberg JB, Kelsey JL, Wilcox HB, McLaughlin JK, Greenberg RS, et al. Oral and pharyngeal cancer and occupation: a case-control study. Epidemiology 1992; 3:300-9.

16. Becher H, Flesh-Janys D, Kauppinen T, Kogevinas M, Steindorf K, Manz A, et al. Cancer mortality in German male workers exposed to phenoxy herbicides and dioxins. Cancer Causes Control 1996; 7:312-21. 
17. Organização Mundial da Saúde. Classificação Estatística Internacional de Doenças e Problemas Relacionados à Saúde. Décima Revisão. São Paulo: Editora da Universidade de São Paulo; 1995.

18. International Agency for Research on Cancer. Tobacco smoking. Monographs on the carcinogenic risk of chemical to humans. v. 38. Lyon: International Agency for Research on Cancer; 1986.

19. International Agency for Research on Cancer. Alcohol drinking. Monographs on the carcinogenic risk of chemical to humans. v. 44. Lyon: International Agency for Research on Cancer; 1988.

20. Breslow NE, Day NE. Statistical methods in cancer research. v. I. The analysis of case-control studies. Lyon: International Agency for Research on Cancer; 1980. (IARC Scientific Publications, 32).

21. Rothman KJ. Epidemiology: an introducion. New York: Oxford University Press; 2002.

22. Vaughan TL, Strader C, Davis S, Daling JR. Formaldehyde and cancers of the pharynx, sinus and nasal cavity: Occupational exposures. Int J Cancer 1986; 38:677-83.
23. International Agency for Research on Cancer. Overall evaluations of carcinogenicity to humans. Lists of IARC evaluations. List of all agentes, mixtures and exposures available to date. http:// www-cie.iarc.fr/monoeval/crthall.html (acessado em 28/Dez/2004).

24. Boffetta P, Gridley G, Gustavsson P, Brennan P, Blair A, Ekstrom AM, et al. Employment as butcher and cancer risk in a record-linkage study from Sweden. Cancer Causes Control 2000; 11:627-33.

25. Pearce N, Matos E. Strategies for the prevention of occupational cancer in developing countries. In: Pearce N, Matos E, Vainio H, Boffetta P, Kogevinas $\mathrm{M}$, editors. Occupational cancer in developing countries. Lyon: International Agency for Research on Cancer; 1994. p. 171-82.

26. Ribeiro FSN, Wünsch-Filho V. Avaliação retrospectiva da exposição ocupacional a cancerígenos: abordagem epidemiológica e aplicação em vigilância em saúde. Cad Saúde Pública 2004; 20:881-90.

Recebido em 24/Jan/2005

Versão final reapresentada em 22/Jun/2005

Aprovado em 06/Jul/2005 